\title{
A Rapid Screening Test for the Diagnosis of Influenza Infection Incubation Period Using Coincidence Analysis of Pulse Waves
}

\author{
Po-Ying Chen ${ }^{1, *}$, Keng-Chang Tu ${ }^{2}$, Shyr-Shen $\mathrm{Yu}^{2}$ and Wen-Kuan Yeh ${ }^{3}$ \\ ${ }^{1}$ Department of Electronic Engineering, National Chin-Yi University of Technology, \\ Taichung 41170, Taiwan; poychen@ncut.edu.tw \\ 2 Department of Computer Science and Engineering, National Chung Hsing \\ University, Taichung 402, Taiwan \\ ${ }^{3}$ Department of Electronic Engineering, National Kaohsiung University, Kaohsiung \\ 811 , Taiwan
}

\section{Abstract}

Viral infections have long been the biggest threat to human survival, and from a medical perspective, the development of noninvasive high-throughput screening methods that target the incubation period to either treat diseases or limit viral spread would be strikingly effective. Using this technology to target viral incubation periods would also be inexpensive to perform. The current study proposes to transform pulse signals into a rapid diagnostic test using "coincidence analysis" in the hope of preventing or reducing the symptoms of viral infections.

The heart plays a critical role in calculating and supplying the needs of all tissues of the body. Pulse waves are pressurized signals in response to heart's calculations and include all phases of the cardiac cycle, which maintains life and provides energy needed to perform tasks. Any small movement gives a corresponding signal to pulse waves. The current study investigated conclusive data on self-limiting infections, such as common cold. We used pulse wave, coincidence analysis technology to capture signals from individuals with common cold during the incubation period and investigated if particular characteristic signals could be applied to influenza during the incubation period. Preliminary work demonstrated that pulse waves could generate signals using this technology that would be worthwhile for future research.

A small amount of analytical data from common cold existed previously. The data structure is based on the idea that a single pulse wave at differing physiological conditions would have slight modifications that would be amplified and presented by various geometrical shapes after extensive data are accumulated. These geometric shapes can then be sliced vertically or horizontally to extract data during different illness stages.

The significance of these findings are impressive; from a personal or a public hygiene perspective, this analytical technology provides many benefits, such as rapid 
and precise decision-making that can be directly visualized or can be analyzed using software programs. Also, this technology also uses futuristic wearable technology that brings practical problem solving to physiology.

Keywords: influenza; pulse waves; incubation period; rapid diagnostic tests; coincidence analysis 


\section{Introduction}

Pulse wave detection via tactile pressure has always been used as a diagnostic method in Chinese medicine. The development of modern medical equipment allows us to understand the roles of and relationships between the nervous system and different organs in the human body.

Current clinical research indicates that the shape of the pulse wave is primarily influenced by pressure created when the heart pulses, arterial wall flexibility, blood vessel resistance, blood viscosity, and other related factors. The arterial system is highly branched; if pulse waves encounter changes in the arteriole transverse surface area (e.g., the arterial tube is narrow, branched, or with sharp ends) or arterial wall flexibility while propagating along the arterioles, a partial reflection of pulse waves is induced. Therefore, taking a pulse by using tactile sensation detects the sum of incoming waves from the heart and all reflective waves after a delay. Thus, pulse waves represent the physiological signals of life that provide different information for sickness, health, and other physiological conditions. The heartbeat is the key to human survival, and the pulse can not only identify physiological conditions but also be used to analyze human behavior. For example, dramatic increases in the heart rate and blood pressure occur during exercise, anxiety, and public speaking. Many physiological states can be detected from the pulse. Thus, pulses can not only reflect individual health status but also determine emotional fluctuations, physical activity, and other physical or environmental changes.

When the human viscera is healthy, the arterial tree resonates at a certain frequency, causing pressure waves to be transduced from the aorta to the arterioles through pulse waves that gradually decrease at capillary joints. When humans become ill, capillaries become blocked, resulting in changes in the aortic and arteriole flexibility and resistance, and as pressure waves propagate to sick regions, abnormal changes are reflected back. Therefore, different reflective waveforms are created when changes in visceral responses occur with changing symptoms, and each reflective wave has the characteristics of the corresponding illness. However, detecting pulses by using tactile sensation requires very sensitive fingers, extensive doctoral training, and is subjective. By contrast, small physiological characteristics stimulated by physical activity are more easily observed on pulse images (i.e., changes in the shapes and intervals of wave fluctuations).

Influenza, also known as nasopharyngitis or acute nasopharyngitis, is very contagious and infects many people each year. Symptoms can be severe and include nasal congestion, runny nose, and fever. Fever results from the severe inflammation of the upper respiratory tract, which can cause dizziness, headache, sore throat, chills, nausea, inappetence, and weakness. Patients with these symptoms are 
usually categorized as having cold or influenza. The "common cold" is a respiratory illness caused by several types of viruses, and although cold is usually present during the early winter, infections can occur in any season. Influenza is caused by only an influenza virus, whereas cold can be caused by several different viruses. Influenza is an acute respiratory infectious disease that dwells in the respiratory tract and is passed among individuals through saliva during coughing or sneezing episodes. The influenza virus is very robust and mutates easily. Therefore, even if someone has previously been infected with influenza, that person can be reinfected, creating situations where huge outbreaks occur, called influenza pandemics. Pandemics often occur during winter and spring, and individuals have a $20 \%-40 \%$ chance of catching the flu each season. Although flu is common, there is no effective method to predict, control, and manage flu at its various stages. Therefore, efficient monitoring and prevention methods are needed to effectively manage influenza.

The autonomic nervous system (ANS) is a self-regulating nervous system. Its function is to regulate, control, and maintain the body at stable and pleasant physiological conditions. The term "autonomic" means involuntary control of nerve activities through consciousness. The ANS controls essential physiological functions, such as heartbeat, respiration, blood pressure, digestion, and metabolism. ANS can be categorized into (1) the sympathetic nervous system and (2) the parasympathetic nervous system. The sympathetic nervous system mainly functions in initiation and excitation, and the parasympathetic nervous system plays a role in resting and rehabilitation.

Recent research studies found that ANS activity may can be measured through the use of using heart rate variability analysis (HRV) analysis, which is an indicator named the heart rate variability (HRV), or so-called the heart rate variability analysis. HRV is a method that used to of measuring the level of consecutive changes in the heart rate changes, and which suggests that in addition to . The idea is that, apart from its own regular electrically-induced beating beats, the heart is also regulated by the ANS. For In the past twenty 20 years, many articles studies have indicated that there is the significant relationship between the ANS and mortality exists that are caused by results from cardiovascular diseases, for example such as sudden death [1] , hypertension [2], hemorrhagic shock [3], and septic shock [4-6],

HRV predict mortality rates related to certain cardiovascular diseases, can be grouped into time and frequency domains, and is most commonly computed as a series of R-to-R peak interval (RRI) times through electrocardiography. HRV usually excludes arrhythmias and other markedly abnormal rhythmic diseases and targets minor heart rate variations with normal heart rhythm. 
In 1965, when Hon and Lee initially described HRV [7], they described a method that could detect changes between pulses in consecutive heartbeats. HRV aims to measure changes in the rate and regularity of a heartbeat; however, many factors can affect HRV, including age, sex, ethnic group, and other pathological factors, such as myocardial infarction, diabetes, and heart failure. In 1981, Akselrod et al. defined the relationship between the parasympathetic nervous system, parasympathetic activity, and heart rate. They believed that the ANS was directly responsible for regulating the heart rate and that it influenced frequency fluctuations, such as arterial blood pressure. Normal heartbeats are affected by ANS regulation and can produce fluctuations that can disappear with maladjustment of the ANS.

When this occurs, the heart rate becomes regular and without fluctuations. These heart rate fluctuations are recognized as ANS heart abnormalities. Appropriate HRV standards were developed by the European Society of Cardiology and the Cardiac Electrophysiology Society of North America to ensure future research directions and included names and terminology, measurement methods, defined physiological and pathological correlations, and appropriate description of clinical applications.

To date, many studies have reported that ANS activity during rest and exercise is significantly different. Ivanovv et al. [8] compared healthy individuals with patients with heart disease and astronauts in severe environments to evaluate differences in HRV parameters while being awake and asleep.

HRV refers to the analysis of heartbeat frequency and the extent of the heartbeat interval distance change. Most studies have indicated that HRV is a relationship between the balances and checks between the ANS and the cardiovascular system. Thus, we can learn from HRV experimental results that different outcomes can be obtained during physiological responses to influenza. Most HRV analyses are extracted directly from changes in RRI times to obtain meaningful physiological signals and are feasible.

Our research team aimed to examine at "the incubation period of influenza" in particular and for personal its physical health and public health issues. As well as operational investigations had also been ongoing for many years and had obtained preliminary results. To analyze physiological information, In addition, our team also took this opportunity to developed a physiological information analysis platform to analyze physiologic information that possessing the following five characteristics, including: (1) easy to operate, ; (2) easy to disassemble and assemble, ; (3) programmable, ; (4) easily to acquire physiological signals, with the opportunity to amplify the signals, ; and (5) non-invasive, these features allow a portable method to obtain can basically be used to do the basic pulse wave measurements. In this 
experiment, we used ANSWatch ${ }^{\circledR}$, a novel multi-functional wrist physiological monitor [9], which was used in order to collect pulse waves at the wrist joint, coincidence analysis was then captured and analyzed. then use the big data analysis to capture 'the flu incubation period' signals, arranging the pulse waves possessing the same baselines at equal intervals were arranged to for overlapping, making causing geometric patterns obtained after the data were as overlapped to create a basis for judgment basis for "the flu incubation period" distinction.

The current study differs from many conventional studies in that it uses a single-pulse amplitude using a large number of stacked numbers that are magnified into different geometric shapes, for which different vertical or cross-sectional cuts can be used to capture criteria to be used in the diagnosis of the "influenza incubation period." We hope to demonstrate that pulse waves can be used in big data analysis and that the development of a "flu signal latency difference" using pulse waves as the backbone can be achieved. The common cold is a viral infection that causes physical discomfort, headache, nasal congestion, and coughing; it is also known as respiratory tract infections, including acute rhinitis, acute pharyngitis, acute laryngitis, and other diseases in medical terms. If cold is not actively treated, it may cause exacerbations, such as acute bronchitis and acute pneumonia.

Some colds may also appear abdominal Bay, diarrhea, and other symptoms, named stomach influenza. The onset of a cold causes many symptoms including (1) sore throat, (2) swallowing difficulty, (3) breathing difficulty, (3) tonsillar hypertrophy, (4) pharyngeal swelling, (5) speaking with a twang, and (6) difficulty in opening the mouth. These physiological responses, in theory, do not generate characteristic pulse wave signals. However, because most of the physiological discomfort experienced in cold acts through the ANS, which can relieve some body movements associated with the illness, capturing characteristic signals with big data analysis of pulse waves becomes feasible.

We used the coincidence analysis method to diagnose patients infected with influenza in preclinical stages as a rapid diagnostic test. This test can determine if a patient with influenza is already infected with the virus by using signals in the big data analysis of pulse waves. This study proposes to use big data pulse wave analysis in a new way. The disease analysis algorithms used in this study are different from many conventional methods used. The overall structure uses a single pulse wave under various physiological conditions that show slight differences. These small differences were amplified using many stacking processes, exhibiting various geometric shapes that can be vertically or horizontally cut to identify all stages of cold or influenza. 


\section{Methods}

\subsection{Participants}

We recruited 38 men and 35 women (aged 20-40 years old) to prevent the impact effect of gender sex and age on HRV testing. Pulses were taken for for the experiment. The time spent sampling the subject's pulse was 0.005 seconds, and the sampling frequency was $200 \mathrm{~Hz}$. The measurements were taken time was set between 22:00- and 23:00 hours coordinated universal time (UTC) daily, for and measured for five minutes each time (considered a "measurement unit") each time as a measuring unit. To be included in the study, Subjects participants subjects were required to complete a normal routine each day without sleep, were not must undergo a day's normal routine without sleep in order to be tested, and they are not allowed to do intense exercise intensely, or to engage in any activity that would result in exercise or any behaviors which may cause them to be in a highly mentally excited state an for onel hour before the experiment, , and were required to wear the subjects will wear the pulse pulse-measuring device in advance before throughout the testing.

Because completely awake and completely asleep states exist, physiological data for each state was captured for 2 minutes. During each measurement, 128 heartbeats were sampled 20 times. Participants were placed into three groups (A, B, or C) with 20 people in each group; none were undergoing medical treatment, and all were in good health. In addition to the aforementioned restrictions, participants were not allowed to stay up late the night before or eat food 1 hour before testing to ensure data objectivity.

\subsection{Experimental equipment and materials}

The hardware included a laptop, a Rhythm Masters wrist monitor (ANSWatchâ), a timer, a scale, and a subjective measuring and recording questionnaire. Software equipment included an ANSWatch Manager Pro data analysis system and an Excel statistical program.

The Rhythm Masters physiology wrist monitor recorded measurements, as shown in Figure 1. If blood pressure and HRV were measured, eight physiological parameters could be obtained in 5 minutes, including SYS, DIA, HR, HRV, LF (NU), $\mathrm{HF}(\mathrm{NU}), \mathrm{LF} / \mathrm{HF}$, and irregular heartbeats. After completion of testing, the abovementioned physiological parameters were displayed on the LCD screen. Up to 50 tests could be stored on the pdf.

The physiology wrist monitor must be worn on the left wrist, and the height should be measured at approximately the position of the heart. Participants were not allowed to move while measurements were being recorded. The monitor was labeled with (a) to designate the front side and (b) to designate the backside so that the reader could recognize the correct position of the monitor. The center line should align with 
the index finger in extension.
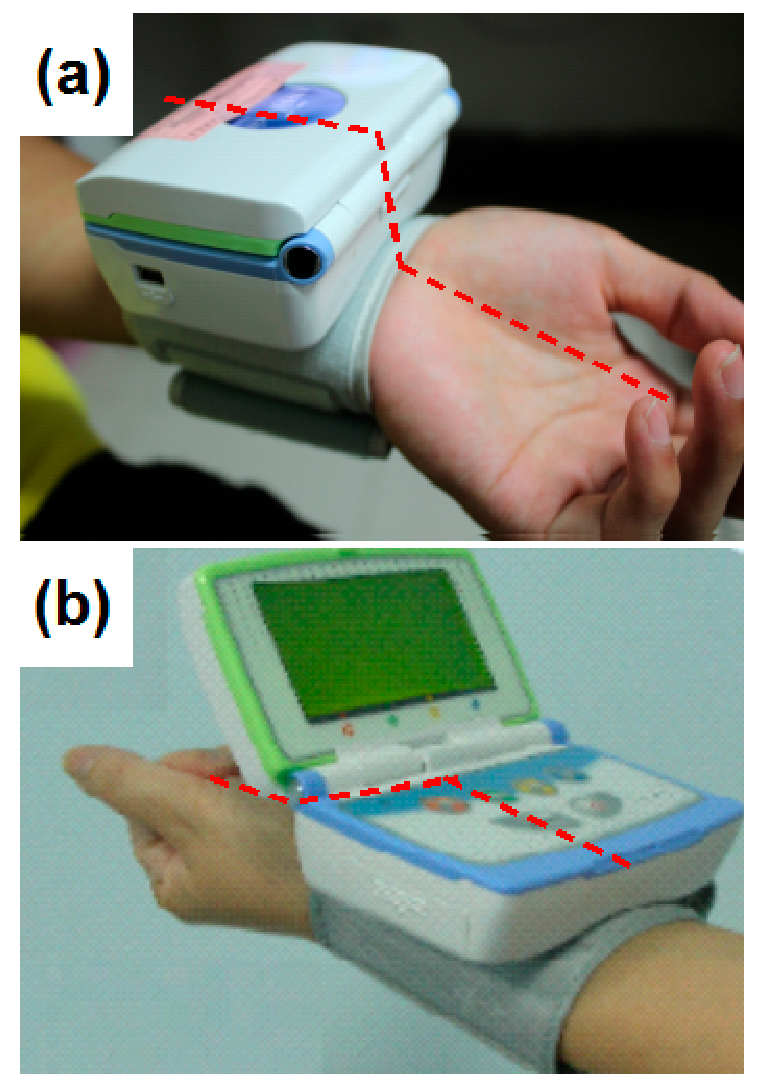

Figure 1 New physiological wrist monitor: Rhythm Masters (ANSWatchâ). This monitor must be worn on the left wrist, and the height should be measured approximately at the position of the heart. Testing lasted 5 minutes, and participants were not allowed to move while measurements were being recorded. (a) the front side of the monitor and (b) the backside of the monitor.

\subsection{Results and Discussion}

\subsection{Influenza Investigation}

"The common cold is a self-healing limiting illness [10-13], the common cold being divided into 'cold' and 'influenza' whereas influenza is a more a severe illness. Overall, the term unlike a common cold, influenza can be associated with more severe symptoms, "common cold" should not be confused with influenza, the latter being more obvious and serious than the former, and also possesses additional symptoms in comparison to the former, such as fever, chills and, muscle muscular pain, and more severe systemic symptoms are more obvious. Influenza can be fatal to those in patients with poor weak immunity (mostly infants, and young children, and the 
elderly people). The difference between the two is that common cold is caused by rhinovirus, whereas influenza is caused by influenza is caused by influenza virus the influenza virus causes influenza [14-18], as a comparison of these illnesses is listed in Table I. Comparison of the differences of the common cold and influenza. Influenza is very contagious, and the viruses usually travels by droplets in the air, contact between people, or spread by being through contact with contaminated items, . and it is a contagious disease. It is Influenza viruses are composed of RNA viruses belonging to within the family Orthomyxoviridae. mucus viral family. Every year, the influenza is popular prevalent during the temperate fall and winter seasons, . Sand serious complications associated with the virus viral infection has led to a higher mortality rate in critically ill patients.
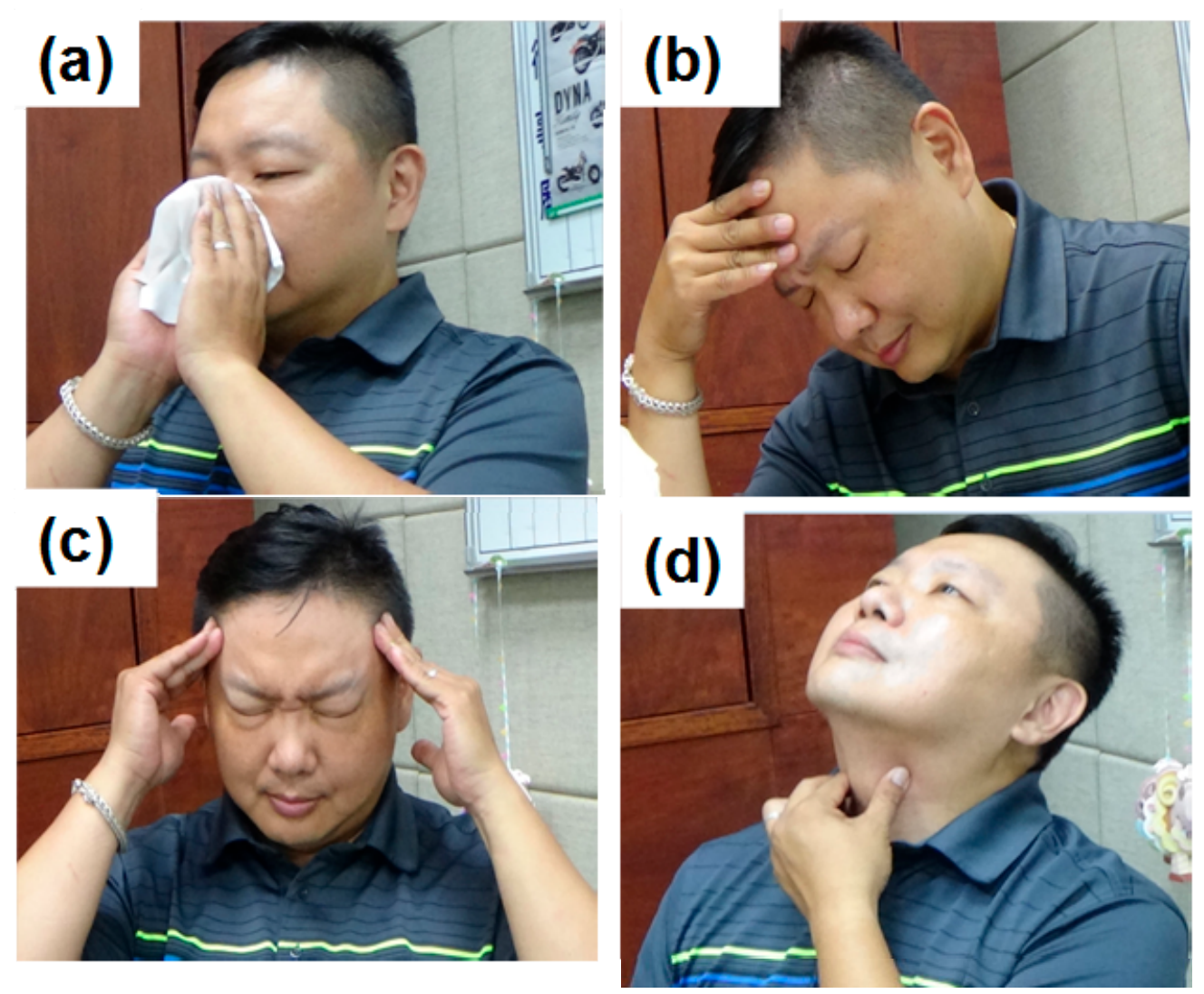

Figure 2. The "flu" can cause physical discomfort, headache, nasal congestion, cough, runny nose, and other symptoms. 
Table I. Common symptoms of cold and influenza illnesses

\begin{tabular}{|c|c|c|}
\hline & Influenza & Cold \\
\hline $\begin{array}{l}\text { Vulnerable } \\
\text { period }\end{array}$ & $\begin{array}{c}\text { October every year to March; October } \\
\text { is one yearly, but spring can be }\end{array}$ & Non-seasonal \\
\hline $\begin{array}{l}\text { Outbreak } \\
\text { process }\end{array}$ & Sudden & Gradual \\
\hline $\begin{array}{l}\text { Systemic } \\
\text { Condition }\end{array}$ & $\begin{array}{l}\text { Severe systemic symptoms with light } \\
\text { respiratory symptoms }\end{array}$ & $\begin{array}{l}\text { No systemic symptoms with severe } \\
\text { local symptoms }\end{array}$ \\
\hline $\begin{array}{l}\text { Fever } \\
\text { Condition }\end{array}$ & $\begin{array}{l}\text { Frequent, and with temperature above } \\
38.3^{\circ} \mathrm{C} \text {, maintained for three to four } \\
\text { days }\end{array}$ & Rare \\
\hline Discomfort & Frequent & Slight to moderate \\
\hline Headache & Severe & Rare \\
\hline Cough & Sometimes severe & Dry cough \\
\hline Sneezing & Occasional & Often \\
\hline
\end{tabular}

\subsection{The importance of latency research}

From a public health management perspective, the incubation period is a critical period before an outbreak occurs, and finding a characteristic signal to identify infection is the primary goal. Currently, the main diagnostic test is a rapid diagnostic blood test and a test measuring viral RNA. These procedures require at least 24 hours, are time consuming, and expensive, which considerably reduce virus prevention efficiency. A more effective rapid diagnostic test is needed. The new test developed should be (1) simple, (2) can be implemented on a large scale, and (3) noninvasive.

Influenza viruses mutate easily, are highly contagious, and often cause epidemics. Figure 3 shows the complete cycle of influenza infection and defines the incubation period. In general, because influenza viruses are transmitted through air, speaking, coughing or sneezing, once a person is infected, the incubation period is short, approximately 1-3 days [19-23]. The onset of clinical symptoms can also be after approximately 7 days. Because infectious viral diseases spread and proliferate in the host, the best strategy to effectively treat this disease is to test as soon as the first evidence of disease is seen. If a person can be tested during the incubation period, they can be quarantined and treated, which is the only effective response to infectious 
viral diseases, such as influenza.

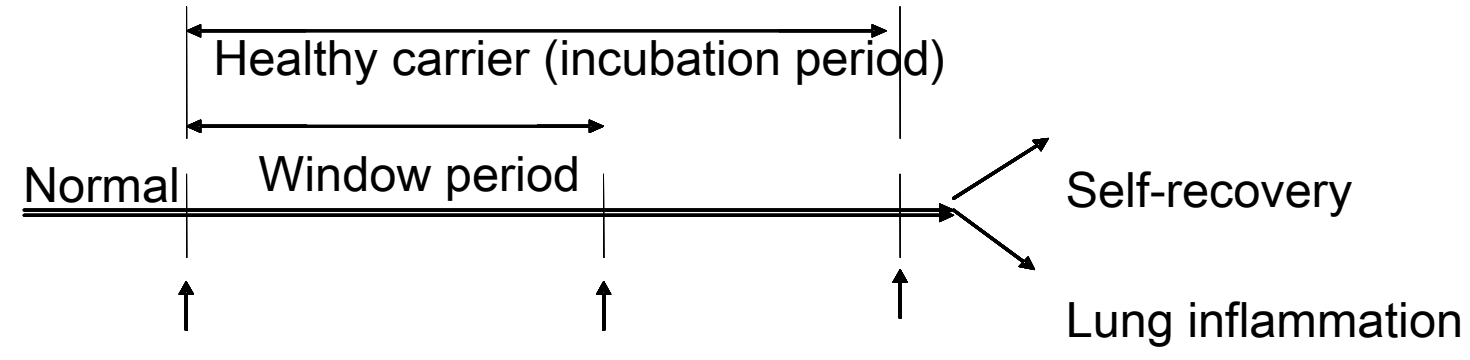

Infected by virusAntibody produced Pathogenesis

Figure 3. The complete cycle of influenza and definition of the incubation period

Most recovery from influenza occurs within 1 week to 10 days [19-23], but some people develop severe lung infections, such as bacterial pneumonia or bronchitis. Differences between influenza infection and other viral diseases and intestinal diseases exist [24-26]. Diarrhea, nausea, and vomiting are often mistaken as influenza. However, influenza usually does not affect the intestines, although approximately $25 \%$ of the infected individuals show signs of nausea, vomiting, and diarrhea [27-32]. Influenza is usually caused by three viral types: influenza A, B, and C [33-39]. Type A occurs once every 10-40 years and can cause worldwide epidemics. Type B has a more limited reach. Type $\mathrm{C}$ occurs less often, and its symptoms are mild; it is a very stable virus. Type A and B viruses mutate frequently. A new epidemic occurs once every couple of years. Malignant virus strains occur in a localized manner and usually occur approximately once every 3 to 15 years. Once infected with influenza, people produce antibodies against the virus, but these antibodies are defenseless against any new strains of the virus. The highest risk groups to have severe complications are those between 6 months and 2 years and those aged 65 years and older. Nursing home residents and patients in chronic care centers are also at a high risk of complications.

\subsection{Study of pulse waves}

Because the pulse is the heart and its accompanying counteractive interactions, a series of actions and reactions causing fluctuations can occur. This process includes (1) the beating of the heart valve induced by the rush of blood sent out by the heart, (2) the beating of arteries caused by the rush of blood sent out by the heart, (3) the rebounding waves of arteries due to the impact of blood sent out from the heart, (4) the backtracking waves produced by blood sent out by the heart, which then runs through the aorta and into smaller arteries, and (5) the expansion and retraction of 
arterial walls during rhythmic diastolic and systolic heartbeats. All pulses can be detected through the artery near the skin surface. These fluctuations cover a complete body cycle; thus, signals of the body and heart get recorded in the process.

A test that is very similar to the measurement of pulse waves is electrocardiography (ECG). ECG records electrical activity of the heart in terms of time through electrodes on the chest skin. The working principle of ECG is that when myocardial cells depolarize during a heartbeat, minimal electrical changes occur on the skin surface, and these small changes are captured as tracing by the electrocardiogram. When myocardial cells are in the resting state, differences in the concentration between positive and negative ions on each side of cells produce a potential difference. When the potential difference of myocardial cells rapidly changes to zero, depolarization occurs, which causes contraction. In the cardiac cycle of healthy hearts, depolarization waves are produced by the sinoatrial node cells and are transmitted through the heart; they are first transmitted to the atrium and then to the ventricles. If two electrodes are placed randomly on each side of the heart, small voltage changes can be recorded, which are displayed on an ECG monitor and traced. ECG recordings can reflect the entire rhythm of the heart, including that the weakest cardiac muscles. However, ECGs have four disadvantages: (1) electrode pads cause inconvenience and discomfort, (2) the machine is large and is not portable, (3) female participants were unable to could not be measured tested in a comfortable environment due to the clothing they wear, and (4) only certain information can be obtained from ECG recordings. By contrast, pulse wave technology does not have these problems, and because it is portable, individuals can have their pulse waves measured daily and continuously until they are infected with influenza and a doctor confirms the disease. Due to previous more basic pulse wave measurements looking at common cold, the corresponding disease symptom information can be used to precisely determine the exact point at which the incubation period starts. If big data stacking analysis is performed on basic measurements that correspond to the incubation period, it is realized that these techniques have a significant application based on the idea that such techniques can transform signals of touch into signals of three-dimensional (3D) vision. These can occur through complete pulse wave capture, and the completion of one-dimensional arrangements and 3D vision stacking. Touch sensation in identifying pulse is not easily understood by many people, machines, or computers, but the transformation touch sensation into $3 \mathrm{D}$ visionary effects is a technique that people, machines, and computers can understand. Figure 4 shows two types of signals related to the heart pulse are displayed, Figure 4(a) shows a type one heart signal using electrocardiography, and Figure 4 (b) shows a type two heart signal using the ANSWatchâ. One can see that the (a) and (b) provide information in 
different forms. With the preliminary data available, data translation from the second type of heart signal (pulse waves) can be performed.
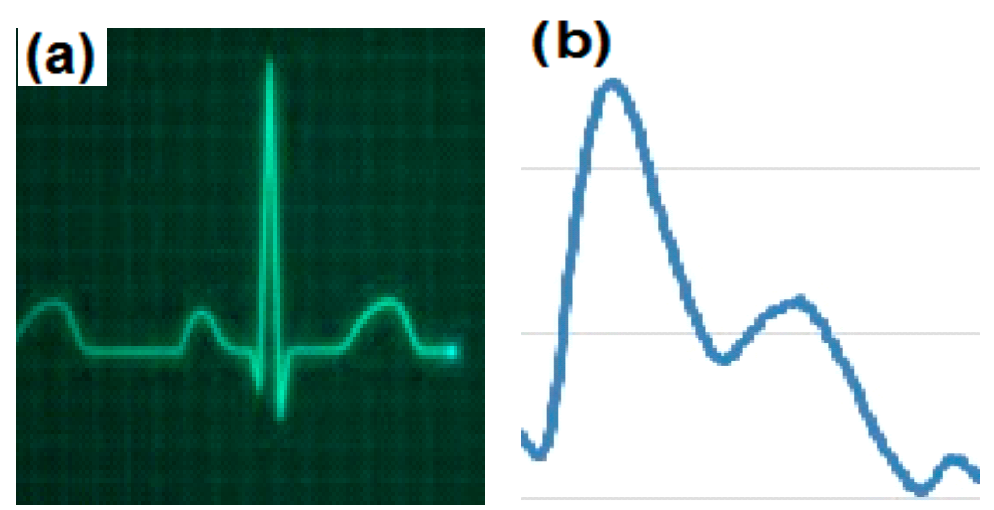

Figure 4. (a) Type one heart signal: electrocardiography (b) Type two heart signal: pulse wave

\subsection{Congruence analysis and the study of pulse waves}

Figure 5 describes the three things completed before capturing heart pulse wave data in our study: (i) pulse wave measurement, (ii) complete pulse wave excision, and (iii) equidistant stacking of pulse waves on the same axis. Completing these three activities presents the entire pulse wave signals in a 3D stereoscopic image. Current studies on pulse wave technology use HRV and physiologically related measurements. However, through careful observation, we found that when RRI times change, pulse wave shapes also change gradually. If we record all complete wave changes and couple those with the effect of big data 3D stereoscopy, a higher number of signals can be displayed. Information garnered from these signals includes pulse wave steepness, amplitude, width, and density intervals. Such information can allow one to create even more physiological signal algorithms.

Figure 5. (a) partial information about basic pulse wave measurements, using 360 pulse waves recorded in 5 minutes. The study used R-to-R peak interval (RRI) times to reveal physiological information. However, as can be seen in this figure, more valuable information is hidden within the data, such as that complete pulse waves represent key physiological signals by taking an entire pulse wave starting from its baseline to find applicable methods. Figure 5 (b) shows the process of capturing pulse waves. After deciding on which sampling number to use, the sampling software locates the position from the top of the R peak, and with that can define the position at which the pulse wave can be captured from the minimum $\mathrm{R}$ peak value. Once the pulse waves are captured, the method of application can be determined. Figure 5 (c) shows a straightforward alignment of captured pulse waves equidistant along the same axis. Such alignment gives interesting results because alignment and stacking create shapes that are similar to that of a mountain. With this method, pulses 
detected using the touch of a finger will be transformed into three-dimensional data, which would allow people, computers, and machines to gain a better understanding of such signals. Many different criteria can be applied to the analysis of "wakefulness and drowsiness" or "uninfected and infected."

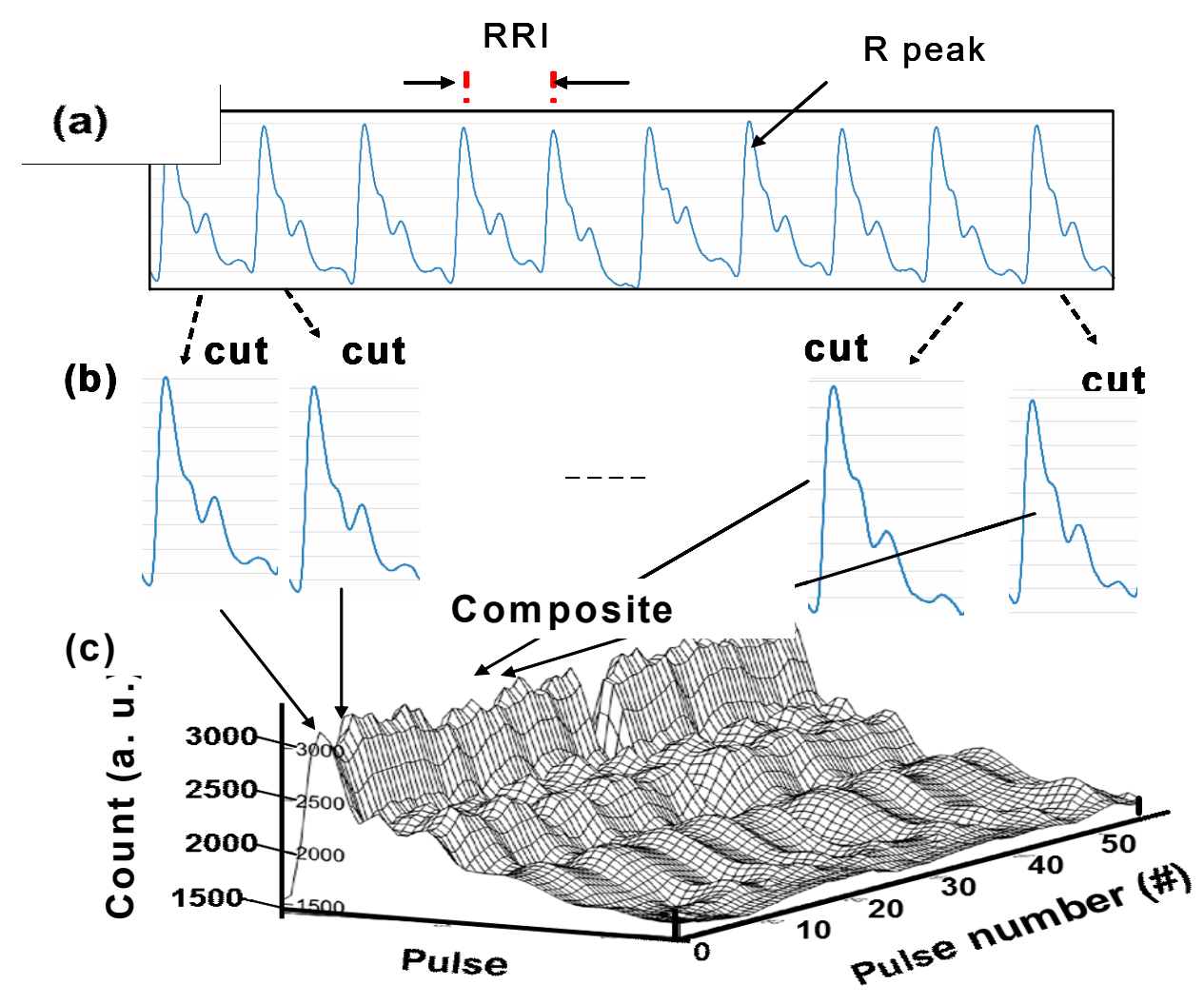

Figure 5 Three actions are completed before computing information captured from pulse waves: (a) pulse wave measurements, (b) complete excision of pulse waves, and (c) equidistant stacking alignment of pulse waves along the same axis.

\subsection{Reliability of the congruence analysis}

Data captured while generating different numbers of pulse waves causes the most concerning issue when performing the equidistant overlapping alignment of pulse waves along the same axis. In case of inconsistent visual effects after alignment, we captured two different numbers from the basic data (50 or 70 pulse waves) and also checked to see if the number of pulse waves captured affected the final result. If both tests passed, then other capture numbers should not interfere with the final results.

Figure 6 shows the capture and exportation of 50 and 70 pulse waves for alignment. This figure indicates that equidistant overlapping of pulse waves along the same axis can transform planarized data into three-dimensional images, which increase and enrich the readability of all the information acquired from pulse waves. 
Such data enrichment presents the HRV of every pulse wave in a more detailed manner. In other words, for all small HRV signals, our team developed functions that allow us to rotate or tilt the overlapped graphics along a certain axis, resulting in equidistant and overlapping alignment along the same baseline and axis and allowing us to perform a detailed inspection of every three-dimensional image. These images indicate that regardless of pulse wave number used to transform the three-dimensional images, the effects are the same.
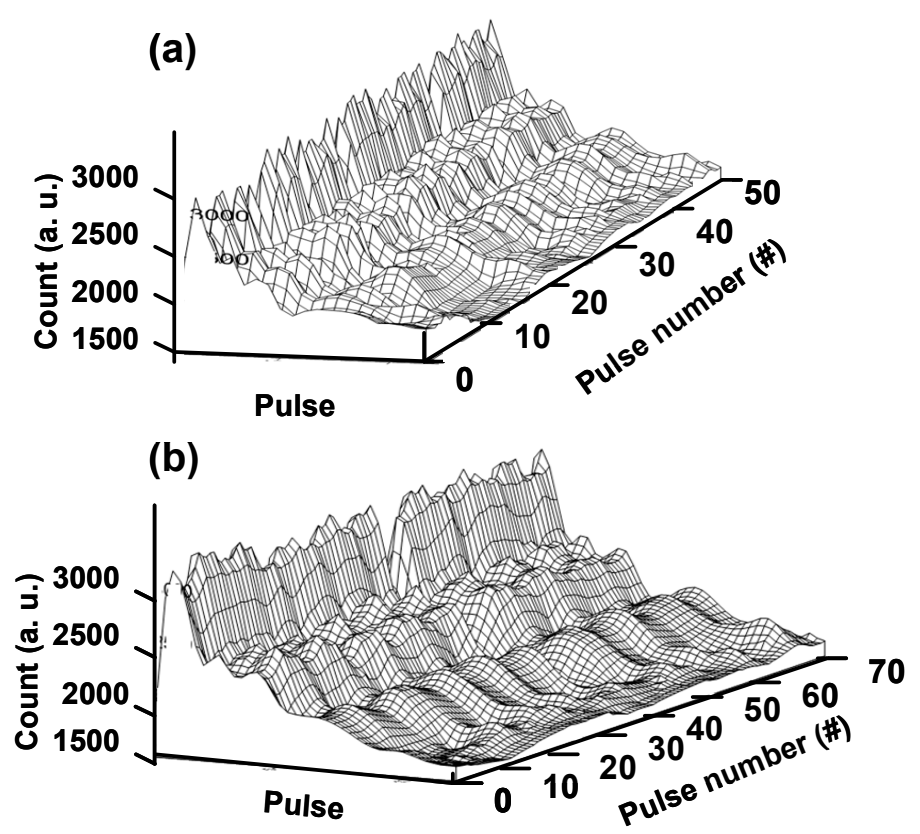

Figure 6. The capture and exportation of basic data from (a) 50 and (b) 70 pulse waves were gathered to perform equidistant and overlapping alignment along the same baseline and axis.

\subsection{Analysis of pandemic influenza pulse waves}

\subsubsection{Analysis of combined double influenza pulse waves}

We then compared the exportation of two neighboring pulse waves from healthy people and patients with influenza. Figure 7 shows that after an influenza diagnosis, all the basic data obtained each day were compared to find common factors. Consecutive pulse waves from the healthy and infected stages of one influenza patient by comparison of graph wave curves were recorded. We found that the pulse wave $\mathrm{R}$ peak amplitude was more uniform, shorter, and wider in the asymptomatic stage compared with wave slopes that were less steep and more complete $(\mathrm{R}, \mathrm{S}, \mathrm{T}, \mathrm{U}$, and $\mathrm{V}$ waves) in the symptomatic stage. Therefore, the total length of each wave was longer, and the $\mathrm{R}$ wave amplitude was higher in the symptomatic stage than in the 
asymptomatic stage. Furthermore, after patients are infected, individual small pulse waves ( $\mathrm{U}$ and $\mathrm{V}$ ) are covered by the following $\mathrm{R}$ wave, which appears in the complete pulse wave curve graphs.

Comparing the exportation of two neighboring pulse waves of "healthy person and flu patient", Figure 7 indicates that after the flu patient has been confirmed by the doctor as being infected by the influenza, all of the basic measured data obtained each day are compared for common evidence. Figure 7 shows consecutive pulse waves from the healthy stage and the infected stage of the same flu patient, through comparison fo the wave curves in the graph, it was found that the pulse wave $\mathrm{R}$ peak amplitude is more uniform and shorter and more fat for the healthy stage, and the slope of the complete waves are less steep and more complete (R, S, T, U, V waves), therefore the total length of each wave is longer, yet on the contrary, the $\mathrm{R}$ wave amplitude average height for the infected stage is higher than that of the healthy stage, also the change in height of the $\mathrm{R}$ wave amplitude is far greater. Furthermore, after the subjects are infected with the flu virus, certain small pulse waves (U, V waves) will be covered by the next $\mathrm{R}$ wave that is to appear in the complete pulse wave curve graphs.

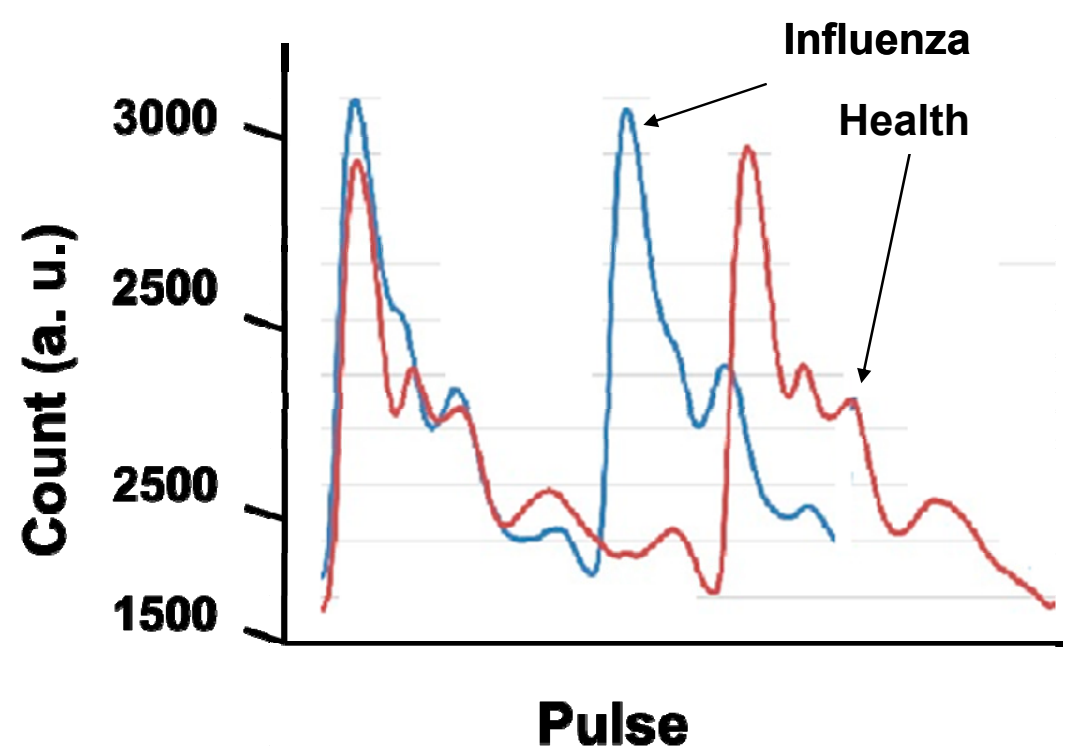

Figure 7. Shapes of consecutive pulse waves recorded during the healthy (red curve-line) and symptomatic stages of influenza (blue curve-line)virus infection in the same infected individuals.

\subsubsection{Analysis of double pulse waves during influenza virus infection}

If we reorganize the data in Figure 7 and separately draw curves of healthy stages and symptomatic stages, as seen in Figure 8 (a), the pulse wave curve during the healthy stage can be observed more clearly. The surface area is also enclosed by 
the connection between the left and right base points of the $\mathrm{R}$ wave (defined as A), and the surface area enclosed by the lowest connection point after reduction of $\mathrm{A}$ is defined as B. Figure 8 (a) shows that the ratio of the R wave area, A, and the reduced area, $\mathrm{B}$, in the asymptomatic stage is $1: 4$, and Figure 8 (b) shows that the same ratio in the symptomatic stage is $1: 2.5$. $\mathrm{R}$ waves represent the left ventricular beat cycle during one heartbeat; therefore, the $\mathrm{R}$ wave surface area $\mathrm{A}$ could be defined as the blood volume pumped out of the heart in every beat. The curve graph in Figure 8 (b) could indicate the time at which the influenza virus already infected the body; the ANS adjusts the heartbeat cycle to a larger left ventricular beat and a faster heartbeat frequency.
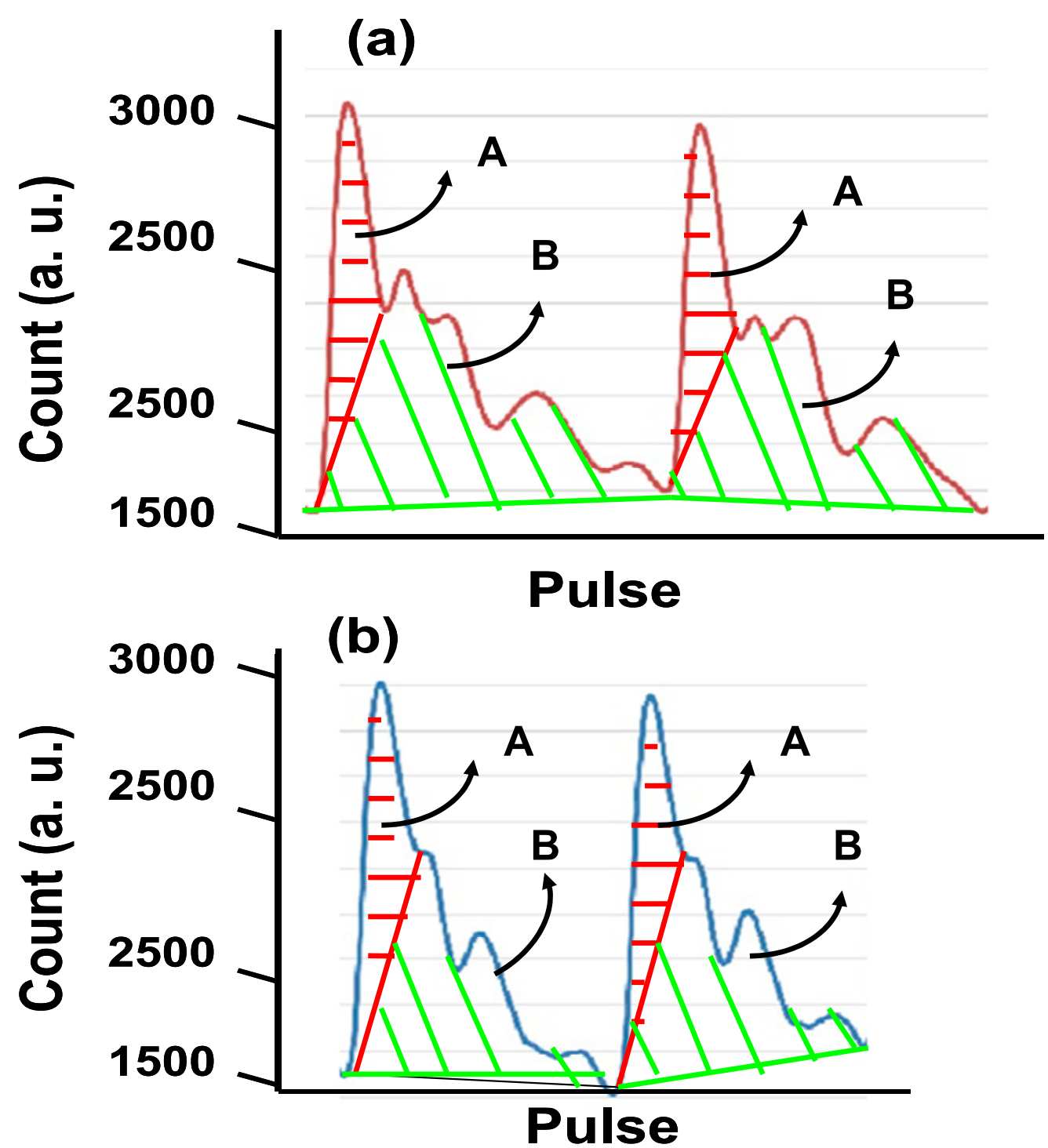

Figure 8. The curve graph of consecutive double pulse waves from (a) the healthy and (b) symptomatic stages of influenza virus infection in the same patient (as in Figure 6). 


\subsection{Congruence analysis of influenza infection pulse waves}

The discussions and presentations in the previous sections have allowed us to understand a few of the pulse waves seen during the asymptomatic and symptomatic stages of influenza infection. Based on the discussion in Section 3.5 and after individuals were diagnosed with influenza infection, our research team exported 60 pulse waves from the daily basic measured data. Figure 9 (a) shows the equidistant overlapping alignment of 60 pulse waves from the individuals in the asymptomatic stage of influenza infection captured along the same baseline and axis. 3D image curve graphs showed that after big data stacking of asymptomatic stage pulse waves, the height decreased portion by portion. By contrast, in Figure 9 (b), data from 60 pulse waves exported during 5 minutes at the symptomatic stage of influenza infection, and 3D image curve graphs revealed a steep, uneven angle distribution and a vertical drop to the base of the curve.
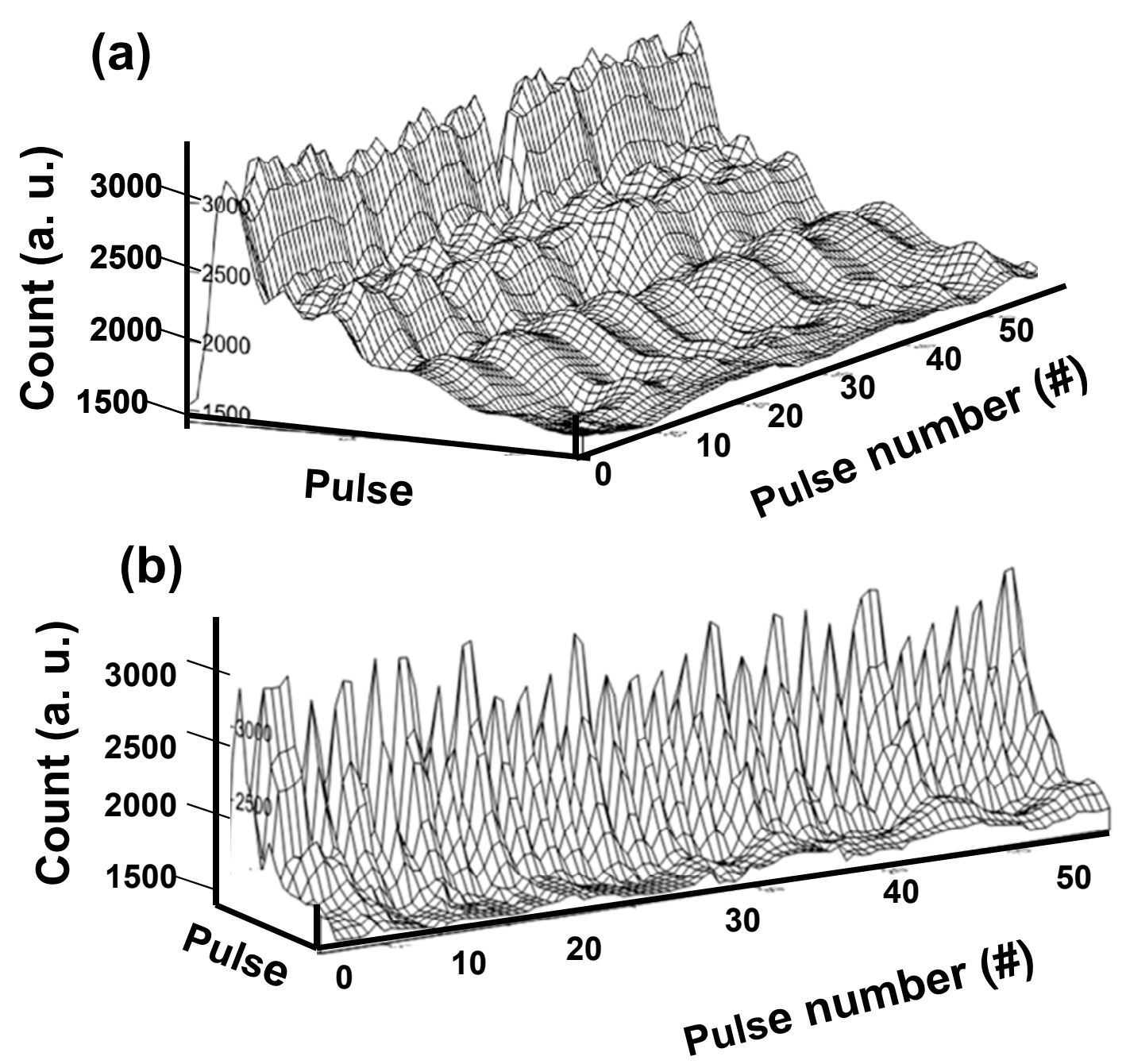
Figure 9. Sixty pulse waves from basic data captured using equidistant overlapping alignment along the same baseline and axis from (a) the healthy and (b) symptomatic stages of influenza virus infection in the same patient (as in Figure 6).

Figure 10 shows the same graph as that shown in Figure 9 with a $45^{\circ}$ rotation along the $\mathrm{Y}$ axis. By performing this inspection, we confirmed that the line produced from 3D images were single straight lines. Figure 10 (a) and (b) shows that the lines of the 3D mountain-like shape were both straight and coherent.

\section{(a)}

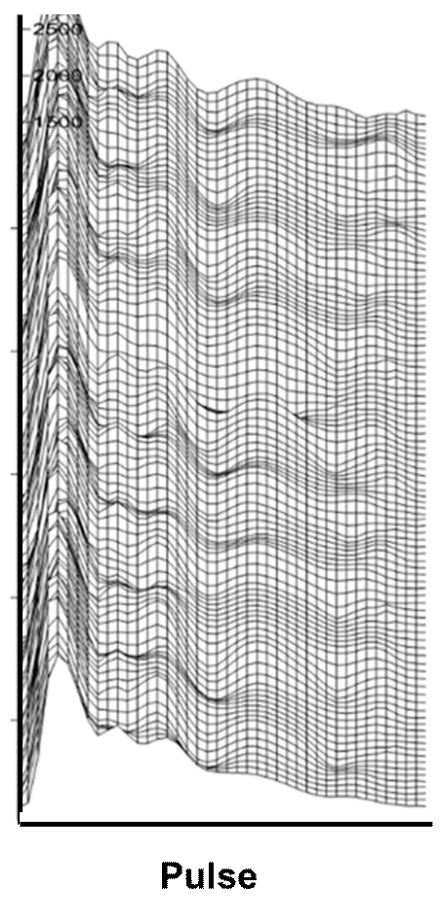

(b)

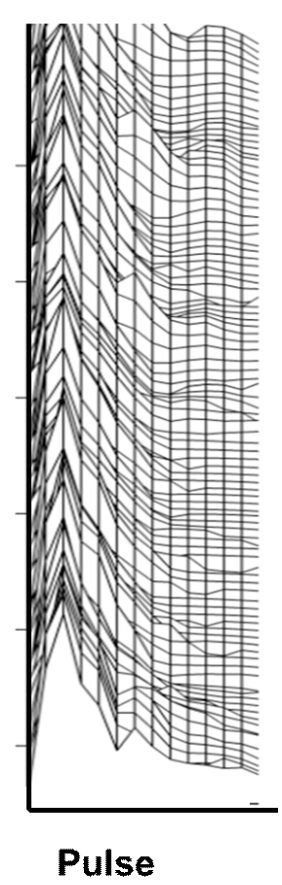

Figure 10. Using the same data as used in Figure 9, data were rotated at $45^{\circ}$ along the $\mathrm{Y}$ axis from (a) the healthy and (b) symptomatic stages of influenza virus infection in the same patient (as in Figure 6)

\subsection{Discussion of cross-sectional graphs of coincidence analysis concerning influenza virus infection}

Figures 10 and 11 confirm that patients infected with influenza virus have easily recognizable differences when comparing healthy versus symptomatic stages of infection. Although these differences are seen at only a few points in only a few of the pulse waves and shapes, five differences are found when these minor differences are analyzed using coincidence analysis (1) amplification, (2) highlights, (3) contrast, (4) 
easy visualization, and (5) easy recording and downloading. The resultant 3D image produced is completely stacked. Figure 11 (a) shows pulse waves from the basic data measured from patients with confirmed influenza infection and who were in the symptomatic stages of infection. The image was obtained from the exportation of 70 pulse waves, with a superimposed arrangement of basic data and $3 \mathrm{D}$ images. The narrowness of the $\mathrm{R}$ wave, and the range, steepness, and shortening of the pulse wave amplitude are pulse waves that identify influenza-infected patients. As shown in Figure 11 (a), we attempted to find physiological signals that were exclusive to the incubation period of influenza is very sensitive. We obtained cross- sectional slices of $3 \mathrm{D}$ images, and when the plane view of the upper section was removed from half of the average height of the mountain-like $3 \mathrm{D}$ pulse wave image, inspection of the image performed.

In Figure 11 (b), we can see that the view obtained after cross-sectioning the 3D image has a large number of closed circles distributed on the edge, which come from differences in 70 exported $\mathrm{R}$ waves. Figure 8, we show that when the body is invaded by a virus, the ANS activates the immune system in an attempt to attack the virus and also performs two other functions: (1) it accelerates the heartbeat cycles and increases the strength of the large left ventricle to pump out blood and (2) it accelerates the homeostatic rate, which is subject to changes in left ventricular strength. These two factors cause the $\mathrm{R}$ wave height and width to become inconsistent during the influenza incubation period, enabling viral counteracting and immune activating waves to occur in a short period. The heart works with these counteracting waves, needing to pump out more blood than usual. The $\mathrm{R}$ wave width becomes narrower to increase the heart rate, and the height of the $\mathrm{R}$ wave increases to increase the amount of blood pumped by the left ventricle.

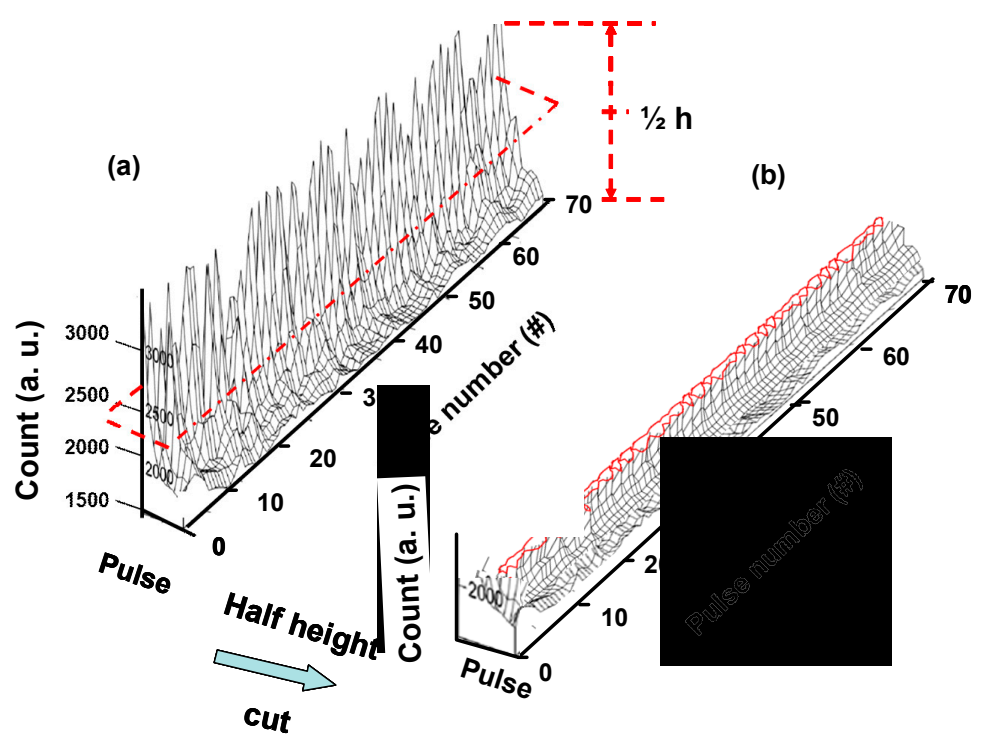

Figure 11. (a) Seventy pulse waves in an overlapping arrangement exported from 
basic data measured in influenza-infected patients. (b) Cross- sections of the upper half of a stereoscopic image, revealing a large number of small close circles distributed over the edge.

\subsection{A rapid diagnostic test to identify the incubation period of influenza virus infection}

The template of this study used basic data collected from influenza-infected patients, who were asymptomatic, and followed them until they became symptomatic. This basic data involved collecting pulse wave data for 5 minutes and then storing the data in a database each day. The data were then traced back from the date of symptom onset because the influenza incubation period is usually 3-4 days before the onset of flu. We investigated the physiological signals of influenza virus infection during the incubation period and used software that could perform (1) equidistant overlapping alignment along the same baseline and axis and (2) stereoscopic cross-sections of data. The first day of the incubation period (Figure 12 (b)) showed differences in cross-sectional images compared with those of the asymptomatic stage. Figure 12 (a)), which included (1) shortening of the complete pulse wave amplitude, (2) shortening of the R wave baseline, and (3) multiple breakpoints at the outer edges of the cross-sectional platform, forming many small closed circles. On the second day of the incubation period (Figure 12 (c)), the three differences of the cross-sectional images were even greater compared with the asymptomatic stage. On the third day of the incubation period, (Figure 12 (d)), the cross-sectional images revealed that the complete pulse wave amplitude was one third of that of the asymptomatic stage; however, the most obvious signal was similarly sized small closed circles throughout the outer edges of the cross-sectional platform. We therefore concluded that the outer edge of the cross-sectional platform is the candidate signal for the influenza incubation period. In Chinese medicine, when palpating the pulse on an individual's wrist, a floating pulse is considered diagnostic for influenza. A floating pulse of Chinese medicine is defined as a strong pulse that is easily taken, but that can be reduced when forceful pressure is applied. Pyrogens cause increased, irregular heart and metabolic rates to generate body heat, causing blood vessels on the body surface to dilate. A relative increase in blood and lymph volumes occurs after an influenza infection, causing the floating pulse to appear. Another pulse classification in Chinese medicine is a "full" pulse. This pulse "full of energy" and is indicated by an increase in breathing and body temperature. When the immune system competes with viruses (vital energy vs. pathogens), especially at the skin surface, capillaries dilate, releasing many immunoglobulins, which increase body temperature and initiates the early stages of a viral infection (the incubation period). Regarding pulse waves, two layers 
of meaning can be inferred: (i) pulse waves with coincidence analysis can be used to detect critical incubation period signals during influenza infection and (ii) pulse waves with coincidence analysis should be measured during the influenza incubation period. The main reasons for the appearance of closed loops on the cross-sectional images of the pulse wave after coincidence analysis are the narrowed width and increased coefficient of variation in every pulse wave. Therefore, the coincidence analysis of pulse waves revealed in this study is a visualized process of the tactile pulse used in Chinese medicine. In other words, the valuable Chinese medical wisdom of tactile pulse responses can be more easily understood using our defined pulse wave technology. Although few people can learn tactile pulse techniques, many more people can understand pulse changes using the coincidence analysis of pulse waves. Future smart medical applications can be developed using this technology.

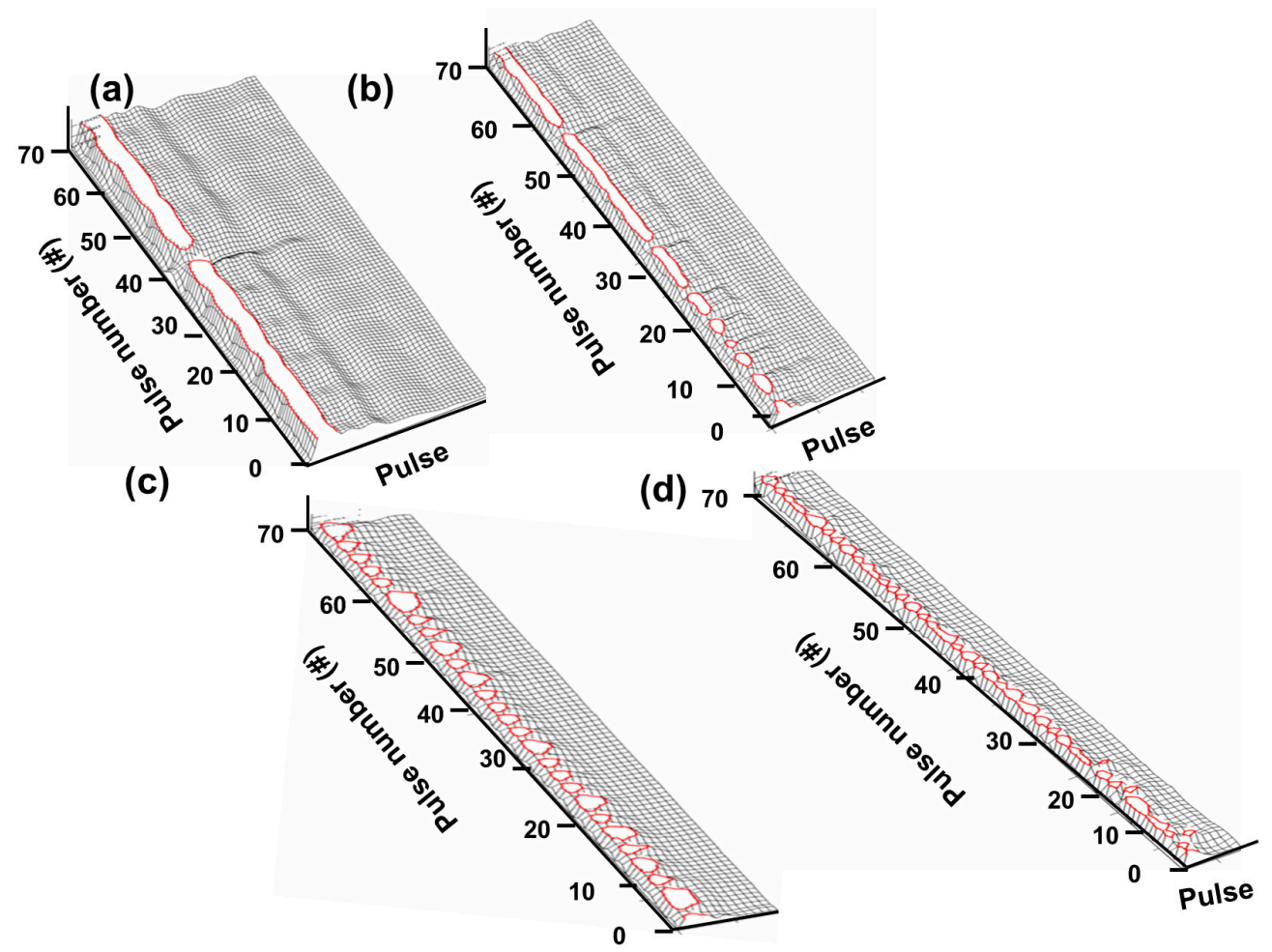

Figure 12. Characteristic signals of the influenza incubation period showing a cross-sectional view of stereoscopic images in patients with influenza infection at (a) the healthy stage, (b) the first day of the incubation period, (c) the second day of the incubation period, and (d) the third day of the incubation period. 


\subsection{The real reason pulse waves are suitable as rapid diagnostic tests for the incubation period of influenza}

With blood as a medium, the pulse signal is carried and mixed into the last wave form. However, blood is a none Newtown fluid (non-Newtonian fluid), and its viscoelasticity changes depending on strain rate, such as temperature, pressure and RBC concentration. Strain rate mainly affects the aggregation and transformation of RBC. At low strain rate, red blood cell aggregates form easily when blood flow has nearly stopped, which results in an increase in frictional resistance in the fluid, and an increase in viscosity. It is not only related to current state of motion, but also the state of motion of fluid in the past. In other words, this type of fluid has a memory effect. Besides the memory effect, the characteristics of a none Newtown fluid(non-Newtonian fluid) include: (i) easy to operate, (ii) short reaction time and (iii) good reproducibility. Since the length scale concerned in the project is often much larger than the distance traveled by moving molecules of fluid, by using a microscopic (molecular) point of view to study the phenomena in fluid, it is not difficult to solve the project's problems. Therefore, all molecules of fluid within a small range of flow field can be considered a fluid element. The scales of fluid elements are much larger than the scales of motion of molecules of liquid. Every fluid element contains numerous molecules. It represents the collective behavior and phenomena of all molecules in the fluid. At this moment, the average characteristics of fluid element, such as density, concentration and speed can be obtained through statistical averages. The macroscopic method, that the space being occupied by fluid is considered as continuous and full of fluid element, is known as the continuum hypothesis. The effects of temperature and pressure on coefficient of viscosity are (1) the coefficient of viscosity of fluid will influence pressure change when affected by temperature. Under normal circumstances, the higher the temperature, the smaller the coefficient of viscosity of fluid, and the pressure of the blood will become higher. (2) For fluid, since the molecules are packed relatively close to one another, the cohesion (attraction) between the molecules is greater. Therefore, cohesion is a major part of the viscous force of the fluid. Hence, when cohesion becomes smaller as the temperature increases, the coefficient of viscosity also becomes smaller. Conversely, the coefficient of viscosity becomes larger when the temperature decreases. The pressure of the pulse will change rapidly with these fluctuations. Hence, the body temperature change will cause the pressure to change during the incubation period, which is the early stage of the influenza virus entering the body, and the pulse waves profile presents a closed-loop pattern after coincidence. However, another signal wave of the heart is electrocardiography (ECG or EKG), and the potential change of ECG is not related to the change in the none Newtown fluid (non-Newtonian fluid) characteristics 
of blood. Therefore, since the body temperature change will not cause the potential to change during the incubation period when influenza virus enters the body, ECG is not a suitable rapid diagnostic test for the incubation period when the influenza virus enters the body.

\section{Conclusion}

To perform physiological analysis, we developed an information platform with the following five characteristics: (1) easy to operate, (2) easy to disassemble and assemble, (3) programmable, (4) easily captures and amplifies physiological signals, and (5) noninvasive. These five characteristics are found using pulse waves with coincidence analysis.

Our particular emphasis was on using this platform to diagnose influenza during the incubation period. We proposed an entirely new set of pulse wave coincidence analysis with algorithms that were different from many conventional methods. The overall structure use single pulse waves in various physiological conditions that could detect slight differences and then amplify the differences so that they can be easily visualized. The data can be easily recorded and combined with other signals and can be presented in different geometric shapes. Longitudinal or cross-sectional cuts can also be performed to capture every viral infection stage.

We found significant differences between asymptomatic healthy individuals and patients diagnosed with early symptomatic influenza infection starting as early as the incubation period. This analytic method has many advantages, including the ability to make precise and fast judgments either visually or by using software programs. It is suitable for future wearable technology. This set of algorithms can complement the medical diagnostics used in traditional Chinese medicine.

\section{Acknowledgement}

We acknowledge funding from the Ministry of Science and Technology under Contract MOST 105-2223-E-390-063-MY1.

\section{References}

1. C.A. Glaser and et al. Medical care capacity for influenza outbreaks, Los Angeles. Emerging infectious diseases, 8(6):569-574, 2002.

2. M.J. Schull, M.M. Mamdani, and J. Fang. Community influenza outbreaks and emergency department ambulance diversion. Annals of emergency medicine, 44(1):61-67, 2004.

3. W.M. McDonnell, D.S. Nelson, and J.E. Schunk. Should we fear "flu fear" itself? Effects of H1N1 influenza fear on ED use. The American journal of emergency 
medicine, 30(2):275-282, 2012.

4. J-P. Chretien, D. George, J. Shaman, R.A. Chitale, and F.E. McKenzie. Influenza forecasting in human populations: a scoping review. PloS ONE, 9(4):e94130, 2014.

5. E. Nsoesie, J. Brownstein, N. Ramakrishnan, and M. Marathe. A systematic review of studies on forecasting the dynamics of influenza outbreaks. Influenza and other respiratory viruses, 8(3):309-316, 2014.

6. Dreifus LS, Agarwal JB, Botvinick EH et al. (American College of Cardiology Cardiovascular Technology Assessment Committee). Heart rate variability for risk stratification of life-threatening arrhythmias. J Am Coll Cardiol 1993; 22: 948-50

7. Hon EH, Lee ST. Electronic evaluations of the fetal heart rate patterns preceding fetal death, further observations. Am J Obstet Gynec 1965; 87: 814-26.

8. Ivanov, I. T., Boytcheva, S. \& Mihailova, G. (1999). Parallel study of thermal resistance and permeability barrier stability of Enterococcus faecalis as affected by salt composition, growth temperature and preincubation temperature. $\mathrm{J}$ Therm Biol 24, 217-227.

9. Taiwan Scientific Corp., http://www.taiwanscientific.com.tw/index.html.

10. R. Perini and A. Veicsteinas. Heart rate variability and autonomic activity at rest and during exercise in various physiological conditions, Eur J Appl Physiol, 2003, Volume 90, pp.317-325

11. D. Balcan, V. Colizza, B. Gon, calves, H. Hu, J.J. Ramasco, and A. Vespignan. Multiscale mobility networks and the spatial spreading of infectious diseases. PNAS, 106(51):21484-21489, 2009.

12. D. Balcan and et al. Seasonal transmission potential and activity peaks of the new influenza $\mathrm{A}(\mathrm{H} 1 \mathrm{~N} 1)$ : a Monte Carlo likelihood analysis based on human mobility. BMC Medicine, 7(1):45, 2009.

13. D. Balcan, B. Gon, calves, H. Hu, J.J. Ramasco, V. Colizza, and A. Vespignani. Modeling the spatial spread of infectious diseases: The GLobal Epidemic and Mobility computational model. Journal of Computational Science, 1(3):132-145, aug 2010.

14. M. Santillana, D.W. Zhang, B.M. Althouse, and J.W. Ayers. What can digital disease detection learn from (an external revision to) Google Flu Trends? American journal of preventive medicine, 47(3):341-347, 2014.

15. M. Biggerstaff and et al. Results from the centers for disease control and prevention's predict the 2013-2014 influenza season challenge. BMC Infectious Diseases, 16(1):357, 2016.

16. R.M. Anderson and R.M. May. Infectious Diseases of Humans: Dynamics and 
Control. Oxford University Press, 1992.

17. E.L. Ionides, C. Bret'o, and A.A. King. Inference for nonlinear dynamical systems. PNAS, 103(49):18438-18443, 2006.

18. C.L. Barrett and et al. Episimdemics: an efficient algorithm for simulating the spread of infectious disease over large realistic social networks. In Proceedings of the 2008 ACM/IEEE conference on Supercomputing, page 37. IEEE Press, 2008.

19. D.L. Chao, M.E. Halloran, V.J Obenchain, and I.M. Longini. Flute, a publicly available stochastic influenza epidemic simulation model. PLoS Comput Biol, 6(1):e1000656, 2010.

20. S. Merler and M. Ajelli. The role of population heterogeneity and human mobility in the spread of pandemic influenza. Proc. R. Soc. B, 277(1681):557-565, 2010.

21. J. Ginsberg and et al. Detecting influenza epidemics using search engine query data. Nature, 457(7232):1012-1014, February 2009.

22. A. Culotta. Towards detecting influenza epidemics by analyzing Twitter messages. In Proceedings of 1st Workshop on Social Media Analytics (SOMA '10), 2010.

23. D.A. Broniatowski, M.J. Paul, and M. Dredze. National and local influenza surveillance through Twitter: an analysis of the 2012-2013 influenza epidemic. PLOS ONE, 12(8):e83672, 2013.

24. H. Achrekar, A. Gandhe, R. Lazarus, Y. Ssu-Hsin, and L. Benyuan. Predicting Flu Trends using Twitter data. In 2011 IEEE Conference on Computer Communications Workshops, pages 702-707, 2011.

25. K. Hickmann and et al. Forecasting the 2013-2014 influenza season using wikipedia. PLoS Comput Biol, 11(5):e1004239, 052015.

26. D.J. McIver and J.S. Brownstein. Wikipedia usage estimates prevalence of influenza-like illness in the united states in near real-time. PLoS Comput Biol, 10(4):e1003581, 2014.

27. T. Bodnar and et al. On the ground validation of online diagnosis with twitter and medical records. In WWW2014 Companion, pages 651-656, 2014.

28. P. Chakraborty and et al. Forecasting a moving target: Ensemble models for ili case count predictions. Proceedings of the 2014 SIAM International Conference on Data Mining., pages 262-270, 2014.

29. J. Shaman and A. Karspeck. Forecasting seasonal outbreaks of influenza. Proceedings of the National Academy of Sciences, 109(50):20425-20430, Nov 2012.

30. J. Shaman and et al. Real-time influenza forecasts during the 2012-2013 season. Nat. Comms, 4, Dec 2013. 318 
31. W. Yang, M. Lipsitch, and J. Shaman. Inference of seasonal and pandemic influenza transmission dynamics. Proceedings of the National Academy of Sciences, 112(9):2723-2728, 2015.

32. R. Gluskin and et al. Evaluation of Internet-based dengue query data: Google Dengue Trends. PLoS Negl Trop Dis, 8(2):e2713, 2014.

33. M.S. Majumder and et al. Utilizing Nontraditional Data Sources for Near Real-Time Estimation of Transmission Dynamics During the 2015-2016 Colombian Zika Virus Disease Outbreak. JMIR public health and surveillance, 2(1):e30, 2016.

34. V. Lampos and N. Cristianini. Tracking the flu pandemic by monitoring the social web. In 2010 2nd International Workshop on Cognitive Information Processing, pages 411-416. IEEE, 2010.

35. C. Corley, A.R. Mikler, K.P. Singh, and D.J. Cook. Monitoring influenza trends through mining social media. In BIOCOMP, pages 340-346, 2009.

36. V. Colizza and et al. Modeling the Worldwide Spread of Pandemic Influenza:

Baseline Case and Containment Interventions. Plos Med, 4(1):e13, 2007.

37. C. Fabrice and et al. Time Lines of Infection and Disease in Human Influenza: A

Review of Volunteer Challenge Studies. American Journal of Epidemiology, 167(7):775-785, 2008.

38. W. K. Oliveira, E. C. Hage, G. O. Penna, R. S. Kuchenbecker, H. B. Santos and W. N. Araujo. Pandemic H1N1 influenza in Brazil: Analysis of the first 34,506 notified cases of influenza-like illness with severe acute respiratory infection (SARI). Euro Surveill;14(42) (2009). 